\title{
Microstructural investigations of EBPVD thermal barrier coatings $\left(^{*}\right)$
}

\author{
L. Lelait $(* *)$, S. Alpérine and C. Diot \\ O.N.E.R.A., Direction Scientifique des Matériaux, B.P. 72, 92322 Chatillon Cedex, France
}

\begin{abstract}
This paper deals with the morphology, constitutive phases and fine microstructures of some EBPVD processed yttria partially stabilized zirconia thermal barrier coatings. This study has been performed by X-ray diffraction and scanning and transmission electron microscopy. It was shown that the coatings studied were constituted of a single metastable tetragonal $t^{\prime}$ phase, as expected if one refers to the well-known non equilibrium paths in the zirconia-yttria phase diagram, and not of a cubic solid-solution, as it is claimed in several papers in the literature. It was also shown that this tetragonality is not easily evidenced by $\mathrm{X}$-ray diffraction because the $\mathrm{t}^{\prime}$ grains exhibit a particular crystallographical texture ( $c$ axis generally parallel to the substrate/coating interface). The relationship between the coating tetragonality, its particular texture and its ability to resist crack propagation is discussed. It is suggested that the former could significantly contribute to the well-known toughness of EBPVD coatings, leading to increased resistance to thermomechanical fatigue.
\end{abstract}

\section{Introduction.}

It is now well established that yttria partially stabilized zirconia thermal barrier coatings (TBC) can lead to significant improvements of turbine engine efficiency and/or "hot components" lifetimes $[1,2]$. The current state-of-the-art consists in applying by air plasma spray (APS) a $\mathrm{ZrO}_{2}-8 \mathrm{wt}$. $\% \mathrm{Y}_{2} \mathrm{O}_{3}$ ceramic coating, on a low pressure plasma sprayed MCrAlY (LPPS), the latter deposited on a nickel base superalloy substrate. However, some evidences have been reported that alternative deposition processes such as electron beam physical vapor deposition (EBPVD) of MCrAlY bond coat as well as zirconia topcoat, could produce coatings with increased thermomechanical resistance [3-7], leading to longer components lives in cyclic oxidation environment. It has also been shown that EBPVD coatings may be processed with a very smooth erosion resistant surface, which is not the case for plasma sprayed coatings. The roughness of the plasma sprayed coatings requires a surface finishing treatment to avoid disturbing the aerodynamic flow around turbine blades. For these reasons, engine manufacturers are finding a renewed interest in EBPVD thermal barrier coatings.

A fairly large number of publications deal with the influence of EBPVD processing parameters on the coating macrostructure, phase composition and thermomechanical behavior $[3,5$, 8-10]. The general consensus is that the EBPVD process leads to the formation of columnar zirconia grains, with a crystallographical texture which varies according to the process parameters [8-10]. The structure of the coatings is described as "segmented" with inter-columnar

(*) Invited paper.

(**) Present address : Electricité de France, Materials Science Department, Centre des Renardières, B.P. 01, Ecuelle 77250 Moret-sur-Loing, France 
voids elongated perpendicular to the ceramic/metal interface. The high thermomechanical strength of these coatings is generally believed to rely mainly on this particular network of microcracks, which is said to be favourable to thermal expansion mismatch stress accommodation.

As far as phase composition of the coating is concerned, several X-ray diffraction studies have been conducted on EBPVD coatings in the zirconia-yttria system. For the classical fully stabilized zirconia (FSZ), $\mathrm{ZrO}_{2}-20 \mathrm{wt} . \% \mathrm{Y}_{2} \mathrm{O}_{3}$ composition, the coating is found, as expected, to be constituted of the cubic yttria solid solution [8]. In the $6-8 \% \mathrm{Y}_{2} \mathrm{O}_{3}$ range, however, most authors observe that the coating is also cubic [5, 8-10], and remains so during high temperature exposures. This result is in fact unexpected and in contradiction with the current knowledge of the $\mathrm{ZrO}_{2}-\mathrm{Y}_{2} \mathrm{O}_{3}$ phase diagram (Fig. 1). Indeed, it is quite conceivable that during the EBPVD process one should not obtain the equilibrium $t+c$ phases, since yttrium diffusion cannot take place. The atoms condensing on the substrate should reorganize in a diffusionless manner (i.e. with very short yttrium atoms displacements) to form the phase with the lowest possible energy. Besides, it is well known [11,12] that upon fast cooling, yttria PSZ spontaneously forms a non-transformable, high yttrium content $t^{\prime}$ phase, which derives from the high temperature cubic solid solution (Fig. 1). It has also been shown that the $c \rightarrow t^{\prime}$ transformation is diffusionless [12] and reversible [13]. Thus, it should not be possible to quench the cubic phase. This implies that the coatings should most probably be made of a tetragonal $t^{\prime}$ and not of a cubic phase, just like in the case of plasma sprayed coatings [1].

This study was initiated to investigate this apparent paradox and also to determine if the fine microstructure of EBPVD yttria PSZ coatings was responsible for their exceptionnally high thermomechanical resistance.

\section{Experimental details.}

The coated substrates were $50 \times 15 \times 3 \mathrm{~mm}^{3}$ platelets of AM1 single crystal nickel base superalloy (Ni-7.9Cr-6.7Co-2.1Ti-5.2Al-2.2Mo-5.7W8.2Ta, wt. \%). Both bond coat and ceramic top coat were EBPVD deposited by Airco Temescal (U.S.A.) using standard processing parameters. The bond coat was a $50 \mu \mathrm{m}$ thick layer of $\mathrm{Ni}-23 \mathrm{Co}-20 \mathrm{Cr}-8.5 \mathrm{Al}-0.6 \mathrm{Y}$ alloy (wt. \%). The ceramic layer had a nominal composition of $\mathrm{ZrO}_{2}-8 \mathrm{wt} . \% \mathrm{Y}_{2} \mathrm{O}_{3}$ and a thickness of 250 micrometers.

The phase structure of the coatings has been analyzed by X-ray diffraction on a diffractometer equipped with a Philips PW1730 simultaneous generator and an horizontal Philips PW1480 goniometer. The radiation used was $\mathrm{Cu} \mathrm{K}_{\alpha}$. A rear graphite foil monochromator was used for signal filtering and peak to background ratio enhancement. The width of the opening slits was $1^{\circ}$, that of the focalisation slits, $0.1^{\circ}$. Data aquisition, storage and processing has been done on a Digital PC 325 microcomputer. Crystallographic textures were analyzed on a $\alpha-\beta$ goniometer using the Schulz method with uncorrected intensities.

The composition of the coatings was determined by EPMA; their macrostructure and morphology was studied by scanning electron microscopy. The fine microstructure of the coatings was studied on a Jeol $4000 \mathrm{FX}$ transmission electron microscope operating at $400 \mathrm{kV}$. For this purpose, transversal thin foils were prepared using a procedure derived from that of King $e t$ al. [14] and fully detailed elsewhere [15]. 


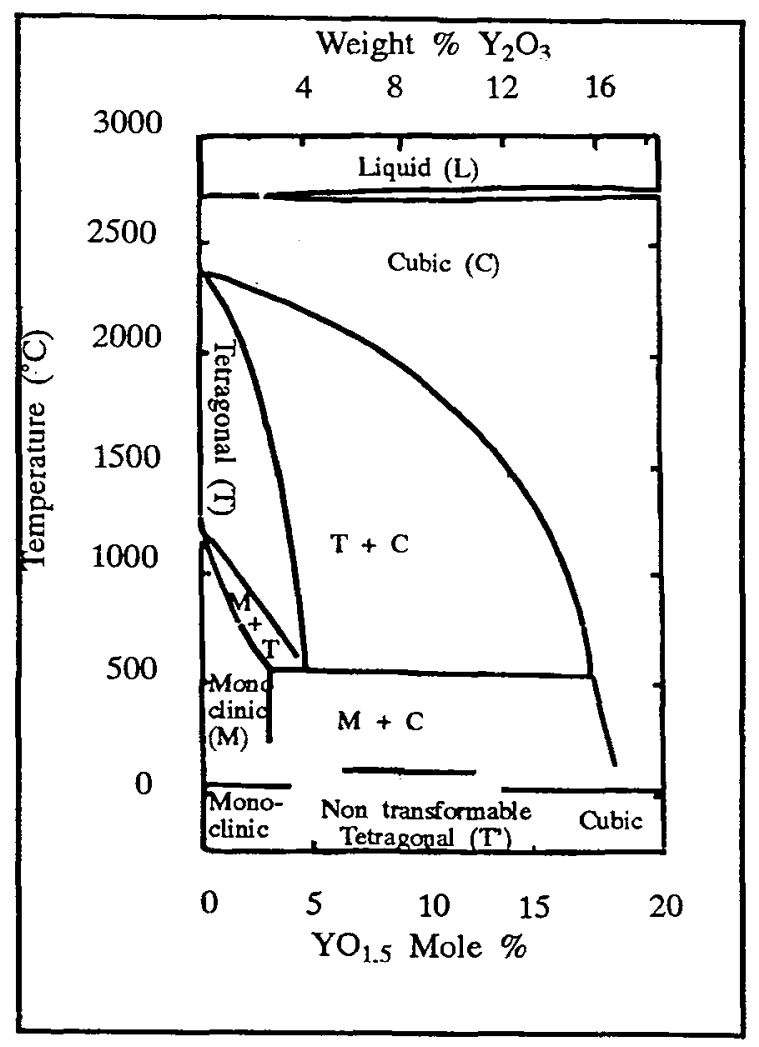

Fig. 1. - Low yttria region of the $\mathrm{ZrO}_{2}-\mathrm{Y}_{2} \mathrm{O}_{3}$ phase diagram established by Scott [18].

\section{Results and discussion.}

3.1 Chemical and Phase COMPOSITION. - The results of chemical analysis performed on the ceramic coatings are summarized on table I. The only foreign element detected is hafnium, which is naturally associated to zirconium. It is to be noted that the composition of the samples very slightly differs from the aimed composition; no important departure from oxide stoichiometry has been recorded. The analyzed yttrium content corresponds to around $8.5 \mathrm{wt} \%$.

Figure 2 shows a typical X-ray diffraction pattern obtained in scanning mode $\left(0.06^{\circ} 2 \Theta\right.$ pseudo-step) on an EBPVD coating. From this single experiment, one might conclude that, as described in the literature [5, 8-10], the coating structure is a cubic solid solution.

However, the displacive character of the $c \rightarrow t^{\prime}$ transformation should again be evoked here. If one refers to other displacive transformations in the zirconia-yttria system, such as the $\mathrm{t} \rightarrow \mathrm{m}$ transformation $[16,17]$, cubic $\mathrm{ZrO}_{2}-8 \mathrm{wt} . \% \mathrm{Y}_{2} \mathrm{O}_{3}$ solid solution should not be retained at room temperature unless :

i) the cubic grains are smaller than a "critical nucleation size" for the $t^{\prime}$ phase (this process being similar to t phase retention in tetragonal zirconia polycrystals) [16];

ii) The cubic lattice sustains high level of compressive stresses which prevent the $c \rightarrow t^{\prime}$ transformation (as it might be the case for instance if large quantities of gas had been dissolved 
Table I. - WDS chemical analysis of the EBPVD ceramic coating.

\begin{tabular}{|c|c|c|}
\hline Elements & $\begin{array}{c}\text { Aimed } \\
\text { composition } \\
\text { atom \% }\end{array}$ & $\begin{array}{c}\text { Measured } \\
\text { composition } \\
\text { atom \% }\end{array}$ \\
\hline $\mathrm{Zr}$ & 30.9 & 30.7 \\
\hline $\mathrm{Y}$ & 2.9 & 3.1 \\
\hline $\mathrm{Hf}$ & - & 0.3 \\
\hline $\mathrm{O}$ & 66.2 & 66.0 \\
\hline
\end{tabular}

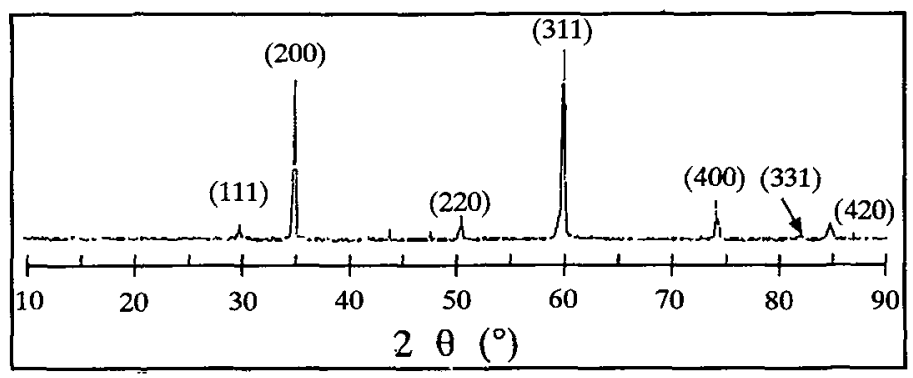

Fig. 2. - X-ray diffractogram of a $\mathrm{ZrO}_{2}-8 w t . \% \mathrm{Y}_{2} \mathrm{O}_{3}$ EBPVD coating, in scanning mode.

into the lattice during the coating formation process).

Figure 2 shows that the diffraction peaks are not particularly broadened, which indicates that the size of the diffracting domains must be larger than $0.1 \mu \mathrm{m}$, thus probably well above the $t^{\prime}$ critical nucleation size; this also indicates that the lattice is not heavily microstressed. Thus, the observation of a quenched cubic solid solution at this stage is a matter of perplexity.

To clarify this point, X-ray diffraction patterns were acquired with a higher angular resolution $\left(0.02^{\circ}\right.$ for $\left.2 \Theta\right)$ and better counting statistics (240 s per step), in specific angular domains where Bragg reflections specific of the $t^{\prime}$ phase (i.e. forbidden by the cubic symmetry) would have been expected. Five such diffraction patterns are reproduced in figure 3 . On every one of them a splitting reveals the actual tetragonal symmetry of the coating. The values of the cell parameters deduced from these diffraction patterns are reproduced in table II, along with the corresponding yttria contents (assessed from the values of $c, a$ and $c / a$, using formula established after the work by Scott [18]). It can be seen that, within the accuracy of the experiment, the measured parameters are independent on the reflection used. The measured yttrium contents are all consistent with the chemical analysis performed on the coating.

In the absence of any crystallographic texture, the left peak over right peak intensity ratios $I_{1} / I_{\mathrm{r}}$ for the splittings in figure 3 should be close to that of the individual peaks multiplicity factor, $m_{h k l}$. The values of $m_{h k l}$ for the reflections displayed in figure 3 are reported in table III along with the theoretical $I_{1} / I_{\mathrm{r}}$ ratio, $r_{\mathrm{th}}$.

It clearly appears that $(h k l)$ reflections with $l>h, k$ are systematically weaker than would be expected according to $r_{\text {th }}$ values. This indicates that the $t^{\prime}$ tetragonal cells are predominantly oriented with their $c$ axis in a plane parallel to the ceramic/metal interface. The recording 

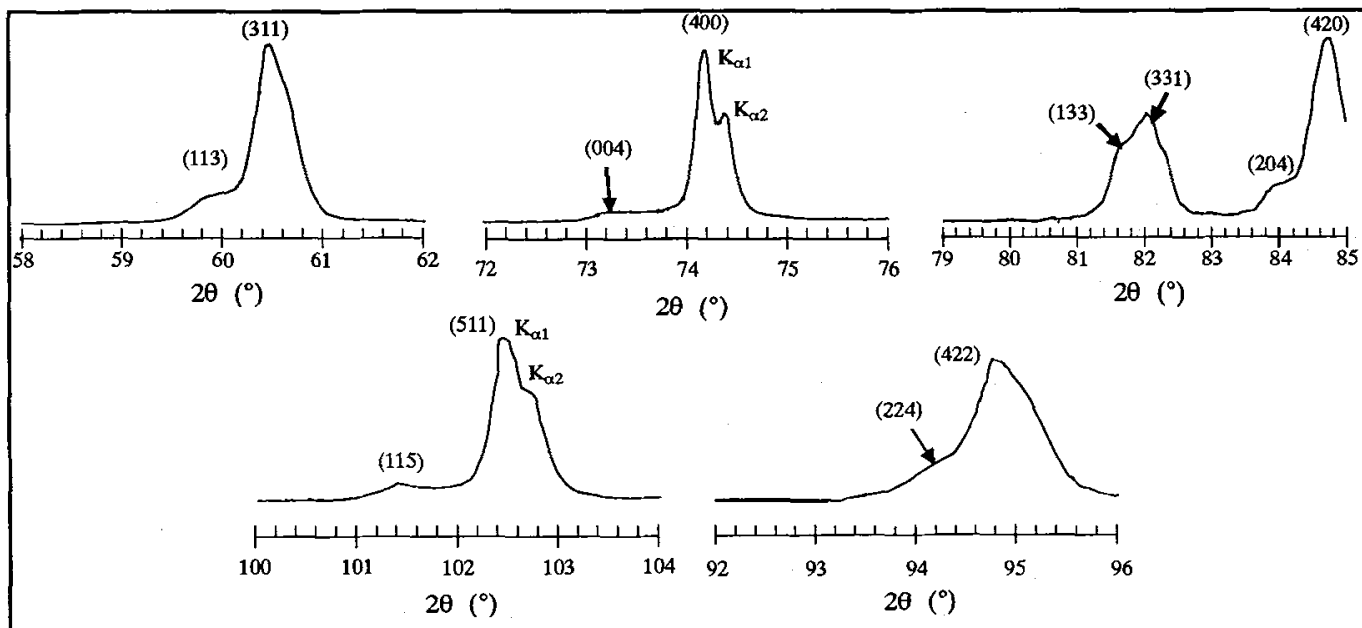

Fig. 3. - X-ray diffractograms acquired in $0.02^{\circ} 2 \Theta$ steps; $240 \mathrm{~s} / \mathrm{step}$; tetragonal splittings are clearly evidenced.

Table II. - Lattice parameters of the $\mathbf{t}^{\prime}$ phase for EBPVD coatings.

\begin{tabular}{|c|c|c|c|c|c|}
\hline 200101 & 8 & (1) & 8601 & 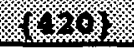 & 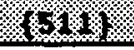 \\
\hline \multirow{3}{*}{ 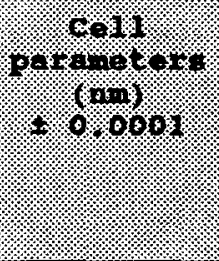 } & . & 0.5111 & 0.5111 & 0.5114 & 0.5111 \\
\hline & 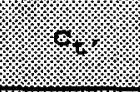 & 0.5167 & $\begin{array}{c}\text { Hardly } \\
\text { measurable }\end{array}$ & 0.5165 & 0.5169 \\
\hline & $7^{2} \times 1.7 \%$ & 1.0110 & - & 1.0100 & 1.0113 \\
\hline \multirow{3}{*}{ 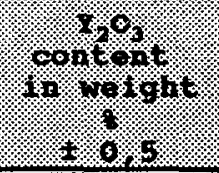 } & 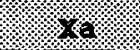 & 7.8 & 8.0 & 8.8 & 8.0 \\
\hline & (1) & 8.7 & - & 9.0 & 7.6 \\
\hline & (2) & 8.1 & - & 8.8 & 7.9 \\
\hline
\end{tabular}

Table III. - Multiplicity factors $m_{h k l}$ of the Bragg reflections of figure 3 and theoretical ratios $r_{\text {th }}$ for the observed splittings.

\begin{tabular}{|c|c|c|c|c|c|c|c|c|c|c|c|c|}
\hline hk1 & 113 & 311 & 004 & 400 & 133 & 331 & 204 & 420 & 224 & 422 & 115 & 511 \\
\hline$m_{n+1}$ & 8 & 16 & 2 & 4 & 16 & 8 & 8 & 8 & 8 & 16 & 8 & 16 \\
\hline$r_{t h}$ & & & & & & & & & & & & \\
\hline
\end{tabular}


of a (100) incomplete pole figure (Fig. 4) confirms that the coatings exhibit a strong (100) crystallographic texture parallel to the ceramic/metal interface. It is worth pointing out here that this particular texture renders the identification of the tetragonal $t^{\prime}$ phase very difficult, since the specific $t^{\prime}$ reflections barely emerge from the background, especially if some $\mathrm{Zr}$ fluorescence were present (which was not the case here because a rear monochromator was used). This might explain why in most published data EBPVD coatings are reported as being constituted of a textured cubic solid solution.

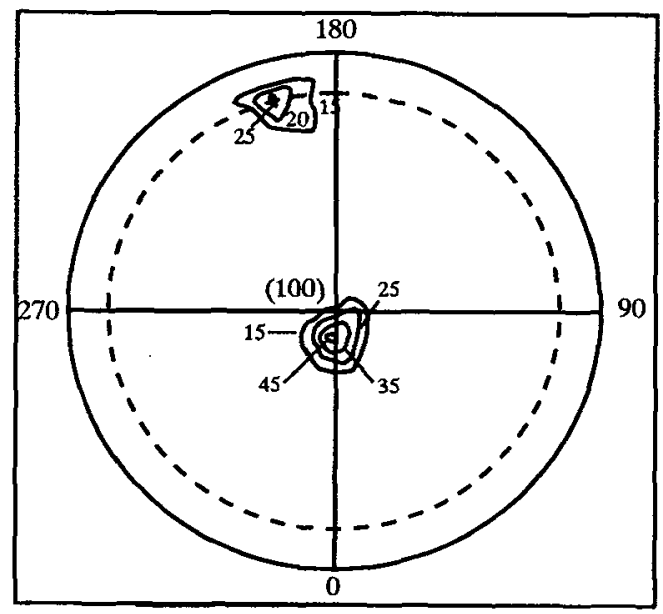

Fig. 4. - (100) incomplete pole figure of an EBPVD coating.

3.2 MORPHOLOGY AND MICROSTRUCTURE. - Figure 5 shows the typical microstructure of EBPVD coatings, as seen by scanning electron microscopy on polished transverse sections: the ceramic growth is strongly columnar and associated with a noticable inter-columnar porosity. The porosity itself is strongly anisotropic and generally oriented perpendicular to the coating surface. At a finer scale (Fig. 6a), the typical width of the zirconia grains is $0.3 \mu \mathrm{m}$, their length being of the order of magnitude of the coating thickness. Electron diffraction also reveals the tetragonal nature of the coating (Fig. 6b).

Besides the inter-grain, finer porosity is observed at the center-line of each columnar grain (Fig. 7). Moreover, very small cuboidal and facetted pores seem to align at regular intervals $(\approx 0.5 \mu \mathrm{m})$, perpendicular to the grain growth axis (Fig. 7).

3.3 THERMOMECHANICAL RESISTANCE OF EBPVD COATINGS. - The high thermomechanical resistance of EBPVD coatings has until now been essentially attributed to the favourable orientation of the intergranular porosity. It is very well known that TBC failure under thermal fatigue condition occurs by the propagation of a fatal macrocrack in the ceramic, parallel to and several $\mu \mathrm{m}$ above the ceramic/metal interface [1,2]. It is then probable that the growth of such a crack, on meeting many orthogonal microcracks, will be substantially slowed down.

Yet, there may be an additionnal explanation for the excellent toughness of some EBPVD coatings. A crack propagating in a zirconia grain will predominantly have to follow one of the easiest cleavage planes. In the type FCC cubic cell these planes are the eight $\{111\}$ planes. 


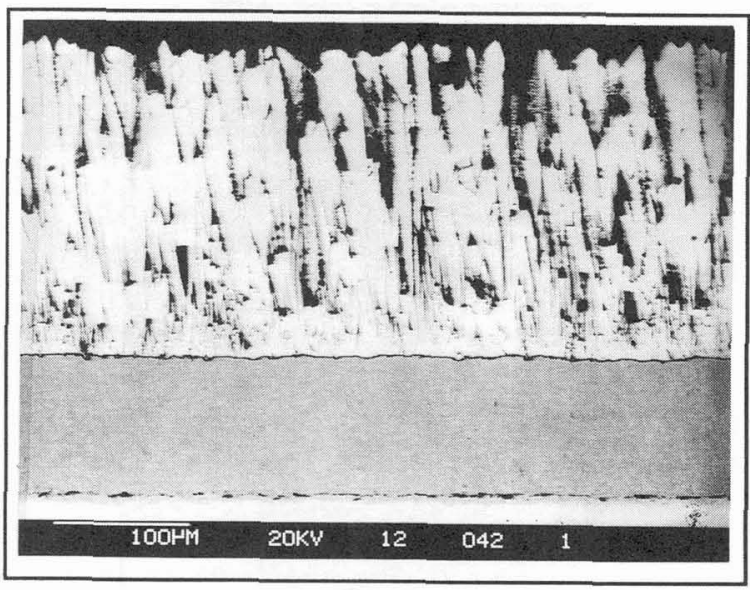

Fig. 5. - EBPVD thermal barrier coating; polished cross section; scanning electron microscope, backscattered electron imaging.

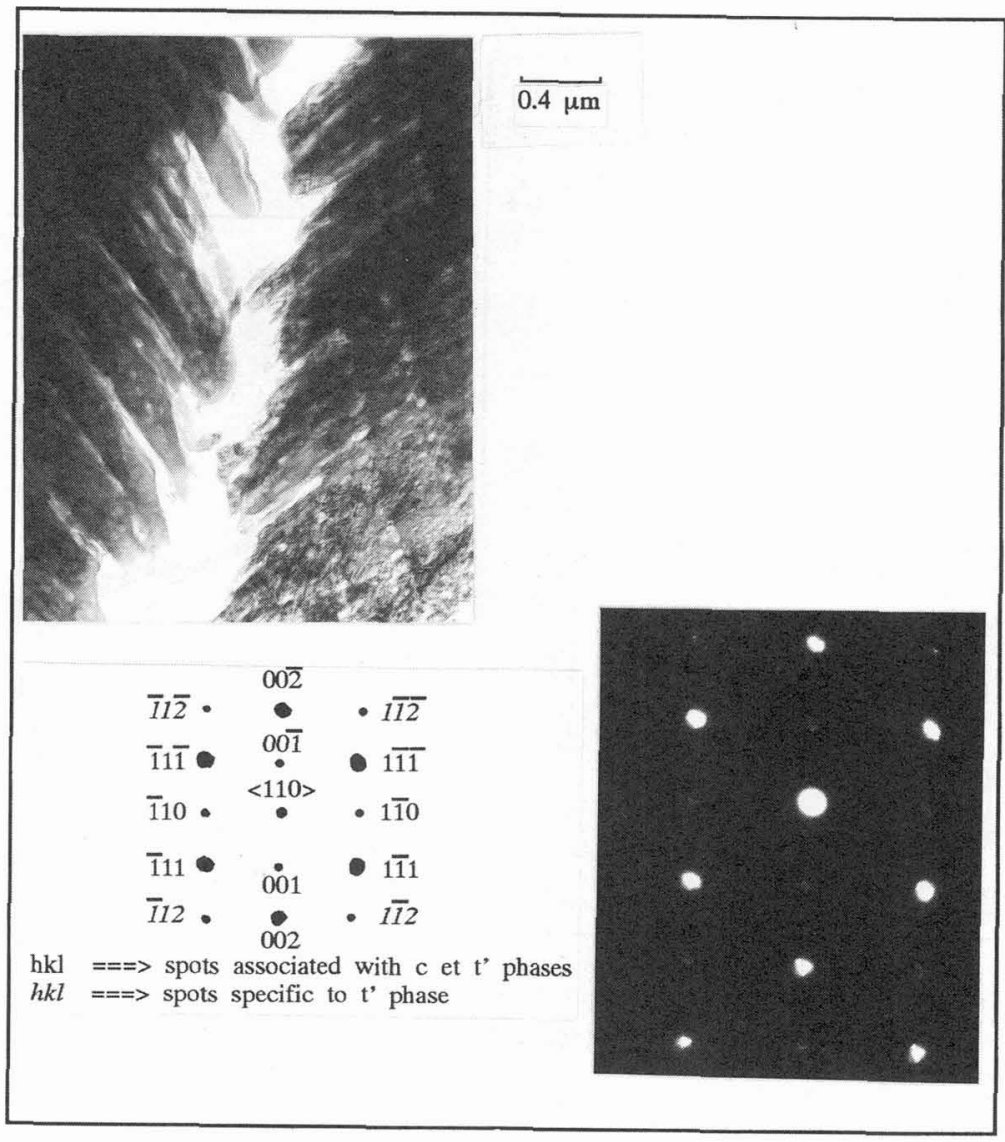

Fig. 6. - EBPVD coating; transmission electron microscopy; a) bright field imaging of columnar grains; b) SAD pattern showing coating tetragonality ( $\langle 110\rangle$ zone axis). 


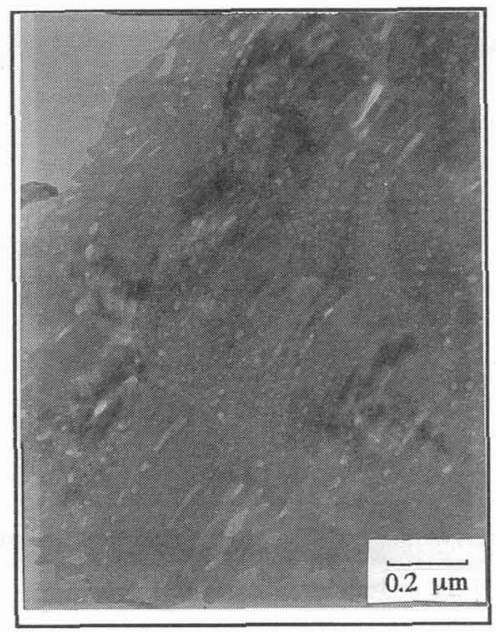

Fig. 7. - Microstructure of EBPVD coating showing pores alignement: TEM, bright field imaging.

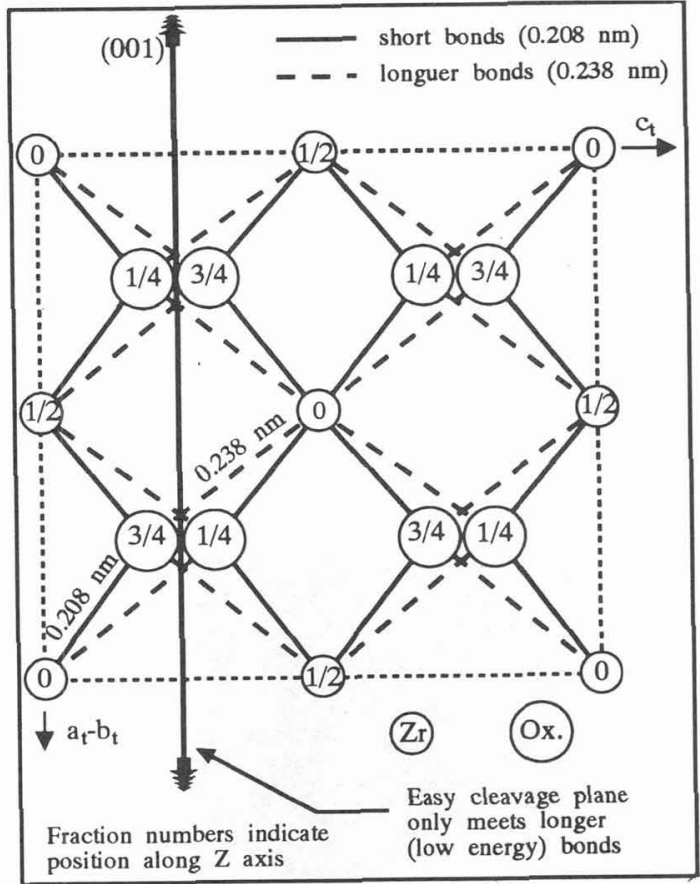

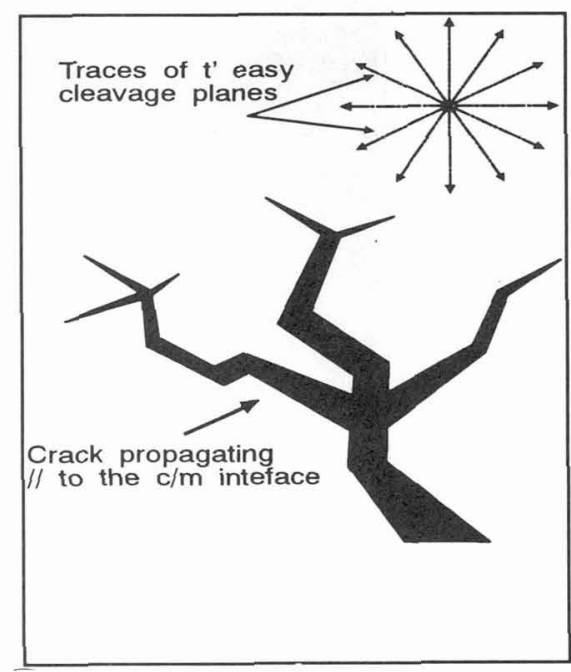

Fig. 9

Fig. 8

Fig. 8. - Schematic representation of a projection of a $t^{\prime}$ tetragonal cell, showing the existence of easy cleavage planes [19].

Fig. 9 - Schematic representation of a crack propagating in the ceramic coating as seen from above; the crack plane seldom meets easy cleavage planes. 
In a tetragonal phase such as $t^{\prime}$, this situation somewhat differs. A projection of the crystallographic cell is shown schematically in figure 8. Atomic bonds are there of two different kinds : shorter bonds (more energetic) and longer bonds (easier to break). One can see in figure 8 that some planes normal to the $c$ axis of the tetragonal cell intercept low energy bonds only. These planes could be the ones along which a crack would propagate with a minimum consumption of energy. If one now considers that the coating has a strong (100) crystallographical texture, it happens that most of the easy cleavage planes, orthogonal to the tetragonal $c$-axis, are also orthogonal to the ceramic/metal interface. A most important consequence is hence that a crack propagating in a plane parallel to this interface is very unlikely to meet easy cleavage planes (Fig. 9). In other words, we propose that the crack propagation, as imposed by the stress system during thermal fatigue, should be slow because :

i) The zirconia coating is of tetragonal symmetry;

ii) It has a marked and well-suited (100) texture.

Of course, in order to ascertain the above proposition, it would be very useful to be able to model and calculate more precisely the crack propagation energies in various $\langle h k l\rangle$ directions of a tetragonal $\mathbf{t}^{\prime}$ cell.

\section{Summary.}

The study of some commercial yttria partially stabilized zirconia EBPVD coatings has revealed that the coatings studied are constituted of a metastable tetragonal $\mathrm{t}^{\prime}$ phase, as expected according to the well-known non-equilibrium paths in the zirconia-yttria phase diagram, and is not a cubic solid-solution, as claimed in several papers in the literature. It has also been shown that this tetragonality is not easily evidenced in X-ray diffraction, because the coatings have a marked (100) crystallographical texture (tetragonal $c$ axis generally parallel to the substrate/coating interface). It is proposed that the combination of these two effects could significantly account for the high toughness of EBPVD coatings, since macro-crack propagation along the ceramic metal/interface would then be slowed down by the scarcity of zirconia easy cleavage planes in this particular direction.

\section{Acknowledgements.}

The authors gratefully acknowledge the contribution of Airco Temescal (U.S.A.) for providing the coated samples and that of T. Ochin at ONERA for X-Ray diffraction measurements. This work was sponsored by SNECMA (France) under contract $n^{\circ} 568260 \mathrm{G}(11 / 22 / 90$ lot 2 étude 1).

\section{References}

[1] Miller R.A., Garlick R.G., Smialek J.L., Ceram. Bull. 62 (1982) 503.

[2] MÉvrel R., Mater. Sci. Eng. A 120 (1989) 13-24.

[3] SCHEN S., Development of improved corrosion-resistance coatings for gas-turbine airfoils, AIRCO TEMECAL, Technical Progress Report réf. DE-AC03-78ET-12292 phase II (1980). 
[4] Fairbanks J.W., Hecht R.J., Mater. Sci. Eng. 88 (1987) 321.

[5] UNAL O., Characterization of thermal barrier coatings formed by electron-beam physical vapor deposition, Proc. of the 47 th Annual Meeting of the Electron Microscopy Society of America (1989).

[6] Toriz F.G., ThakKer A.B. and GuPTA S.K., Surf. Coat. Technol. 39/40 (1989) 161-172.

[7] Sheffler K.D., GUPTA D.K., J. Eng. Gas Turbines Power 110 (1988) 605.

[8] JAMES A.S., MATTHEWS A., Surf. Coat. Technol. 41 (1990) 305-313.

[9] FANCEY K.S., MatTHews A., J. Vac. Sci. Technol. A4 (1986) 2656.

[10] James A.S., FANCEY K.S., MATthews A., Surf. Coat. Technol. 32 (1987) 377-387.

[11] LANTERI V., Ph.D., Case Western University, (Cleveland, OH, U.S.A., Mai 1986).

[12] Lanteri V., Chaim R., Heuer A.H., J. Am. Ceram. Soc. 69 (1986) 258.

[13] Lelait L., Thèse, Université d'Orsay (Novembre 1991).

[14] King W.E., Peterson N.L., Reddyj F., Proc. Int. Cong. on Met. Corrosion (Toronto, June 1984) p. 4.

[15] Lelait L., Alpérine S., Diot C., Mévrel R., Mater. Sci. Eng. A 120/121 (1989) 475-482.

[16] Anderson C.A., Greggi Jr. J., Gupta T.K., Adv. Ceram. 12, Am. Ceram. Soc. 194 78-85.

[17] Rhule M., Claussen N., Heuer A.H., Adv. Ceram. 12, Am. Ceram. Soc. (1984) 352370.

[18] SCOTT M.G., J. Mater. Sci. 10 (1975) 1527.

[19] Michel D., Mazerolles L. and Perezyjorba M., J. Mater. Sci. 18 (1983) 1618-1628. 